\title{
The influence of cutting conditions and cutting tool geometry on the atomistic modeling of precision cutting
}

\author{
Angelos P. Markopoulos*, Nikolaos E. Karkalos, Kalliopi-Artemi L. Kalteremidou, Andreas \\ Balafoutis and Dimitrios E. Manolakos
}

Section of Manufacturing Technology, School of Mechanical Engineering, National Technical University of Athens, Heroon Politechniou 9, 15780, Athens, Greece

\begin{tabular}{|c|c|}
\hline ARTICLE I N F O & A B S T R A T T \\
\hline $\begin{array}{l}\text { Article history: } \\
\text { Received } 6 \text { January, } 2015 \\
\text { Accepted } 3 \text { April } 2015 \\
\text { Available online } \\
3 \text { April 2015 } \\
\text { Keywords: } \\
\text { Molecular dynamics } \\
\text { Simulation } \\
\text { Nano-machining } \\
\text { Cutting forces } \\
\text { Morse potential }\end{array}$ & $\begin{array}{l}\text { In this paper a molecular dynamics simulation of nano-metric cutting of copper with a diamond } \\
\text { tool is presented. MD simulations require the determination of the interaction of the involved } \\
\text { atoms through a function of potential for the materials involved in the analysis and the accurate } \\
\text { topography of the studied area, leading to high demand of computational time. The models } \\
\text { presented are taking into account the cubic lattice of copper, test two different potential } \\
\text { functions and at the same time control the computational cost by introducing small models at } \\
\text { realistic cutting conditions. This is realized by a novel code developed and allows focusing on } \\
\text { the influence of several processes and modeling parameters on the outcome of the simulations. } \\
\text { Models with and without thermostat atoms are investigated and the influence of cutting } \\
\text { conditions and cutting tool geometry on chip morphology, cutting forces and cutting } \\
\text { temperatures are studied. }\end{array}$ \\
\hline
\end{tabular}

\section{Introduction}

Nanotechnology is considered nowadays one of the most technologically advanced scientific fields. Several applications of nanotechnology are already included both in special and everyday products and the interest in further developing this modern field of science is increasing. As far as nanoscale manufacturing processes are concerned, the thorough understanding of the parameters of these processes and the physical mechanisms involved are needed in order to increase their efficiency and capabilities. Considering that the amount of experimental data and the capabilities of measurement techniques are nowadays rather insufficient, numerical methods have been developed for the investigation of nano-machining processes. Perhaps the most used method for modeling manufacturing processes is the Finite Elements Method (FEM) (Markopoulos, 2013). More specifically, in the field of micromachining, several works pertaining to FEM simulations have already been published; a review

* Corresponding author. Tel : +302107724299

E-mail addresses: amark@mail.ntua.gr (A. P. Markopoulos) 
can be found in (Markopoulos and Manolakos, 2014). However, the Molecular Dynamics (MD) method is one of the most used numerical methods in nano-scale simulations. It is considered more appropriate than other numerical methods which have been used for machining process simulations, as macro-scale methods include the assumption of a continuum; in nano-scale this assumption is not helpful and instead a proper modeling of inter-atomic forces is required. Although MD method was developed over six decades ago for general atomistic simulations (Metropolis et al., 1953; Alder and Wainwright, 1957), it is currently used in many engineering fields. MD is also applied to simulate several precision manufacturing processes and nano-scale cutting (Komanduri and Raff, 2001; Oluwajobi and Chen, 2010).

Early MD simulations in nano-cutting processes were carried out by Belak and Stowers (1990) and Belak et al. (1993), who investigated the nano-cutting process of copper and silicon both for 2D and 3D configurations for several depths of cut and rake angles. Later, Ikawa et al. (1991) studied the effect of the minimum depth of cut and the ratio of the depth of cut to the radius of curvature in the specific cutting energy, the surface deformation and the chip formation mechanism. Inamura et al. (1992) presented a model for the transformation of an atomistic model to an equivalent continuum model for the nano-cutting process. Maekawa and Itoh (1995) studied the effect of friction between a copper workpiece and a diamond cutting tool. Kim and Moon (1995) studied the cutting mechanism of copper and aluminum using a 2D model and Morse potential and investigated the behavior of each material in two different depths of cut and cutting speeds. Komanduri et al. (1998) conducted simulations on ultra high precision cutting processes, investigated the effect of the cutting tool shape in this process and also (Komanduri et al., 2000) investigated the effect of the crystal direction, the cutting direction and the rake angle in an aluminum nano-cutting simulation. Ye et al. (2003) carried out simulations with large negative rake angle values and a copper cutting tool in a copper workpiece nano-cutting process. They investigated the effect of different cutting speeds in the chip forming mechanism and machined surface quality. Furthermore, Pei et al. (2006) investigated the effect of potential function on the cutting forces during nano-cutting.

As the influence of the temperature during the cutting process is significant in every scale of simulation, several efforts to include a proper temperature calculation in MD nano-cutting simulations have to date been made. Rentsch and Inasaki (1994) included a model for temperature regulation in their study concerning ultra high precision cutting processes. Isono and Tanaka (1997) studied the effect of workpiece temperature in the nano-cutting mechanism and surface roughness. Cai et al. (2007) studied the temperature field in a silicon workpiece nano-cutting process. Dziedzic et al. (2008) included a Nosé-Hoover thermostat model in a study of the cutting tool degradation and were able to predict the local temperature fields in their system. Romero et al. (2013) analyzed the temperatures profile and the thermal phenomena occurring in a nano-cutting machining process, using a classic orthogonal cutting model. Romero et al. (2014) also integrated a thermostat model in an MD study of tribological shearing. Furthermore, Zhu et al. (2014) carried out simulations of copper nano-cutting process and investigated the effect of the temperature of the workpiece on the cutting forces. Li et al. (2015) studied the effect of the grinding speed in the temperature profile.

In this study, several investigations regarding nano-machining processes using the MD method are conducted. The effect of a variety of machining parameters proven to be of major importance also in conventional cutting process such as depth of cut, cutting tool speed, cutting tool shape and rake angle are examined using an orthogonal nano-scale model. Furthermore, the effect of the use of temperature boundary conditions in the MD model by adding thermostat atoms to the model is also investigated in several cases.

\section{The Molecular Dynamics method}

The basic aspects of the Molecular Dynamics method are the equation of motion, the potential function, the inter-atomic forces calculation, the numerical time integration of the equation of motion using appropriate numerical schemes and the boundary conditions. Potential functions such as LennardJones or Morse potential function are used for the calculation of the inter-atomic forces. The choice of 
the appropriate potential function is crucial as each potential is often more suitable for a certain category of materials whose nano-scale behavior it can simulate to a sufficient degree. Complex potential functions, such as the embedded-atom model (EAM) potential, are also extensively used in MD simulations but often, simpler and less time consuming potentials can also produce sufficiently accurate results.

The Lennard-Jones potential is suitable for those metals whose behavior can be described using the so-called "hard-sphere model". The "6-12" formulation of the Lennard-Jones potential that is often used is expressed as:

$$
V_{i j}=4 \varepsilon\left[\left(\frac{\sigma}{r}\right)^{12}-\left(\frac{\sigma}{r}\right)^{6}\right]
$$

where $\varepsilon$ is the depth of the potential well, $\sigma$ is the distance at which the inter-particle potential is zero and $r$ is the distance between two atoms. The Morse potential is also appropriate for simulations of fcc metals and is frequently used in MD simulations due to its relatively low computational cost. The formula for the Morse potential is given by:

$$
V_{i j}=D\left(e^{-2 a\left(r_{i j}-r_{e}\right)}-2 e^{-a\left(r_{i j}-r_{e}\right)}\right)
$$

where $r_{i j}$ is the distance between the atoms $\mathrm{i}$ and $\mathrm{j}, r_{e}$ is the equilibrium bond distance, $D$ is the well depth and the parameter $a$ is related to the 'width' of the potential function and controls the magnitude of the potential well. Parameters $D$ and $a$ have the dimensions of reciprocal distance and energy, respectively. The equation of motion is solved in order to calculate the displacement, velocity and acceleration of each atom in the study. Using the potential function, the forces exerted on each atom can be calculated by differentiating the potential energy function and then used in the differential equation system, e.g. the Newton equations of motion, to calculate the displacement, velocity and acceleration of atoms.

There are three main methods for the calculation of atomic interactions (Rapaport, 2004): the allpairs method, the cell subdivision method and the neighbor-list method. These methods are used to find which atoms are to be included in the inter-atomic forces calculation and often constitute the most timeconsuming part of an MD code.

An appropriate numeric time integration scheme is required to be chosen for the time integration of the equation of motion. The key factor in this choice is that the method should not require the calculation of inter-atomic forces multiple times in each time step, leading to a rather inefficient code in connection to the computational time required, unless the accuracy of the results is radically improved. The most preferable integration schemes in MD simulations are the Verlet algorithm, the Leapfrog method and predictor-corrector schemes.

Eventually, the choice of the initial and boundary conditions is equally decisive for an MD simulation. The structure of each material present in the simulation, i.e. fcc or bcc lattice, must be properly defined as well as the initial velocity and acceleration for each atom. The choice of the initial velocities in the system is performed in regard to the conservation of the total momentum in the system. In MD nano-cutting simulations two basic categories of boundary conditions are often imposed: boundary conditions on the motion of atoms of the workpiece and boundary conditions for the regulation of temperature in the workpiece and sometimes in the cutting tool. Several methodologies for the regulation of the temperature are used in $\mathrm{MD}$, namely Berendsen algorithm, Velocity rescaling, Nosé-Hoover algorithm just to name some. These algorithms differ in the way they enable the adjustment of the temperature to the desired temperature.

\section{Simulation parameters}

An orthogonal nano-cutting model is used in every simulation in this paper. The main parameters used in each simulation are presented in Table 1 and shortly described afterwards. The simulations were carried out with a generic in-house Molecular Dynamics software. 
Table 1. Simulation parameters

\begin{tabular}{|c|c|}
\hline Configuration & 2D-cutting \\
\hline Potential function & Lennard-Jones, Morse Potential \\
\hline Workpiece dimensions ( $\AA$ ) & $143 \times 35$ \\
\hline Workpiece material & Copper (fcc) \\
\hline \multicolumn{2}{|l|}{ Number of workpiece atoms } \\
\hline Cases without thermostats & Cases with thermostats \\
\hline $684 \quad$ (Newtonian) & $576 \quad$ (Newtonian) \\
\hline \multirow[t]{2}{*}{ (boundary) } & 116 (boundary) \\
\hline & 108 (thermostats) \\
\hline Tool dimensions $(\AA)$ & $60.06 \times 3.08$ \\
\hline Number of tool atoms & 120,240 (in some cases) \\
\hline Tool material & Diamond (supposed C) \\
\hline Tool edge & sharp \\
\hline Tool rake angle (degrees) & $0,10,20,30$ \\
\hline Depth of cut $(\AA)$ & $8,10,12,14,15,20,30$ \\
\hline Cutting speed $(\mathrm{m} / \mathrm{s})$ & 123,246 \\
\hline Cutting direction & $-\mathrm{X}$ \\
\hline Bulk temperature (K) & 293 \\
\hline Integration scheme & Leapfrog \\
\hline Integration time step (fs) & 10 \\
\hline Total simulation time & Varying according to the cutting speed \\
\hline Total cutting length $(\AA)$ & 130 \\
\hline
\end{tabular}

The main cutting tool and workpiece characteristics are depicted in Fig.1. The x-axis is considered to be in the horizontal direction and the $y$-axis in the vertical direction. Thus, the cutting tool will move towards the -x direction. The copper workpiece has dimensions of $143 \AA$ x $35 \AA$ and consists of 800 $\mathrm{Cu}$ atoms in an fcc lattice. The workpiece material consists of three distinct zones: the Newtonian, the boundary and thermostat atoms. The main purpose of the use of the boundary atoms is to prevent the Newtonian atoms from an unwanted displacement and subsequently preserve the shape of the workpiece. These atoms are placed at the left edge and the bottom of the workpiece. The Newtonian atoms are able to move according to the Newton's equation of motion under the effect of inter-atomic forces. Finally, two layers of thermostat atoms are also added to the model in order to regulate the temperature in the workpiece. Thermostat atoms remain at a constant temperature, equal to the initial bulk temperature of the workpiece, namely $293 \mathrm{~K}$, and contribute to the heat dissipation. The arrangement of all types of atoms is also depicted in Fig.1.

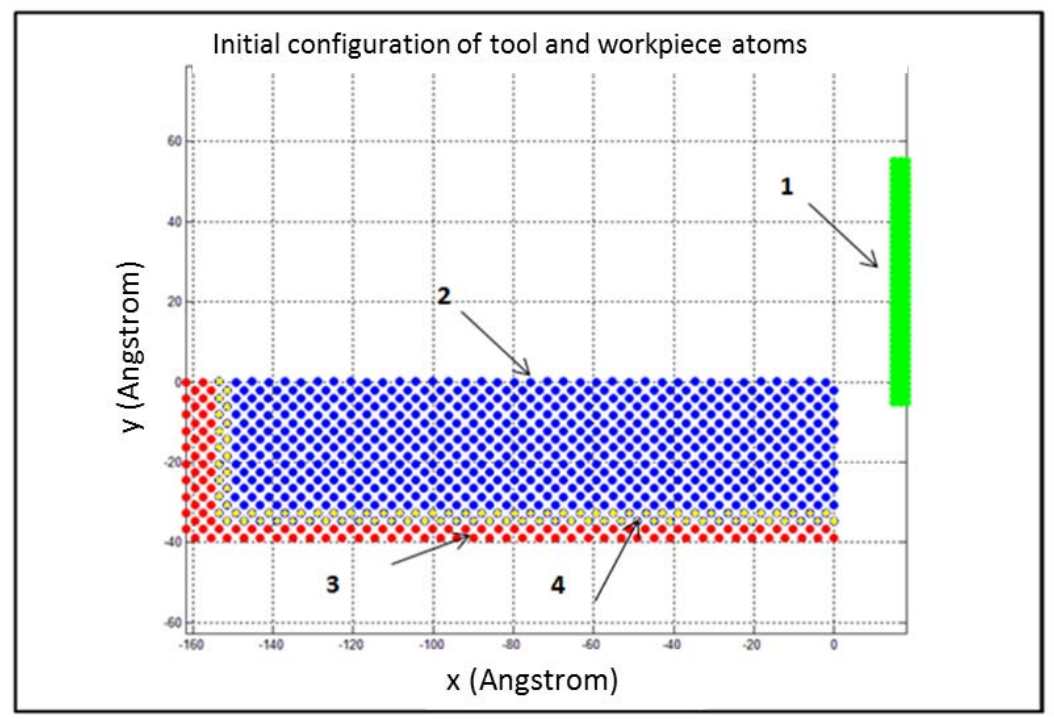

Fig.1. Geometric characteristics of the workpiece and the cutting tool and different types of atoms used in the simulations (1: cutting tool; 2: workpiece; 3: boundary atoms; 4 : thermostats) 
The cutting tool has a rectangular shape, consists of 120 atoms and has dimensions of $60.06 \AA \mathrm{x}$ $3.08 \AA$. The cutting tool material is a single-crystal diamond. The tool is considered perfectly rigid and a varying rake angle between 0 and $30^{\circ}$ is used. In some cases, a tool with a different morphology, consisting of 240 atoms, is used. The starting position of the tool is 2 Å right from the copper workpiece in every case; cutting tool contains no boundary or thermostat atoms.

Both the $\mathrm{Cu}-\mathrm{Cu}$, within the workpiece atoms, and $\mathrm{Cu}-\mathrm{C}$, between cutting tool and workpiece atoms, interactions are modeled using the Morse potential. The Morse potential parameters were selected from the relevant literature (Girifalco and Weizer, 1959) and are presented in Table 2.

The Leapfrog numerical integration scheme is used for the time integration of the Newton equations of motion. The time step value of $10 \mathrm{fs}$ was found to be sufficient for the time integration, after conducting several test simulations with a time step range of 1-20 fs. The total cutting length is $130 \AA$ and it is common for every case. For the regulation of the temperature, a velocity rescaling algorithm, that enables the recalculation of velocities when a certain temperature difference is observed, was employed.

Table 2. Morse potential function parameters

\begin{tabular}{llll}
\hline & $D(\mathrm{eV})$ & $a\left(\mathrm{~A}^{-1}\right)$ & $r_{\mathrm{e}}(\mathrm{A})$ \\
\hline $\mathrm{Cu}-\mathrm{Cu}$ & 0.3429 & 1.3588 & 2.866 \\
$\mathrm{Cu}-\mathrm{C}$ & 0.0870 & 5.1400 & 2.050 \\
\hline
\end{tabular}

\section{Results and Discussion}

The mechanisms of chip formation as well as the effect of several parameters like depth of cut, rake angle, cutting speed, tool size and shape, potential function and temperature regulation on the nano-cutting process, were investigated throughout this paper. Two series of different model cases were conducted, more specifically, cases including thermostat atoms and cases without thermostat atoms. In the first series of the investigations, cases with three different depths of cut, namely 10, 15 and $20 \AA$, four different rake angles, namely $0^{\circ}, 10^{\circ}, 20^{\circ}$ and $30^{\circ}$, two different cutting speeds, 123 and $246 \mathrm{~m} / \mathrm{s}$, two different cutting tool shapes and two different potential functions were evaluated. In the second part of the investigations, the use of thermostat atoms for cases with four different cutting depths, namely 8, 10, 12 and $14 \AA$ and two different potential functions were conducted. Observations on the effect of these parameters on chip formation mechanism, machined surface quality, cutting forces and temperature distribution within the workpiece, were conducted.

\subsection{Cases without thermostat atoms}

\subsubsection{Description of the chip formation mechanism}

In Fig. 2 the results of the simulations with a cutting tool with $0^{0}$ rake angle and $15 \AA$ depth of cut, for three different time steps are shown. At first, it was observed that the workpiece material is being deformed in front of and around the area where the cutting tool moves, as it happens in conventional cutting processes. The workpiece material far from these areas is barely affected by the movement of the cutting tool. As the cutting process continues further, the chip moves along the tool surface. At the end of the process, a chip is formed in front of the cutting tool. It was found that, with an increase of the depth of cut, the deformation of the workpiece material increases and lower surface quality and voids occur due to the more intense interactions between atoms. Furthermore, more anomalies are observed in the chip shape and the irregularity of the machined surface increases.

\subsubsection{Effect of tool rake angle and depth of cut}

The increase in rake angle influences the chip formation, as the ratio of $F_{y} / F_{x}$ decreases and an improved surface quality is observed. Note that cutting forces ratio is indicative of friction between cutting tool and the formed chip. In Fig. 3, the chip morphology is shown for three different rake angles, namely $10^{\circ}, 20^{\circ}$ 
and $30^{\circ}$, for depth of cut $10 \AA$. By increasing both the rake angle and the depth of cut, a rougher surface is obtained, but it is clearly more regular than in cases with zero rake angle. By increasing the rake angle further, fewer voids are observed within the workpiece and the chip height is bigger. Fig. 4 depicts various combinations of depth of cut and rake angle. In Fig. 5 (a) and (b) the tangential and the normal cutting force, respectively, versus the depth of cut, for various rake angles, is plotted. It is obvious that both cutting forces increase linearly with an increase in the depth of cut.

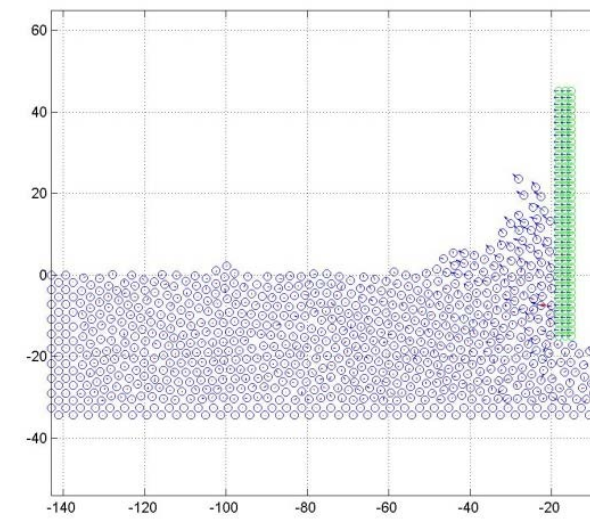

(a)

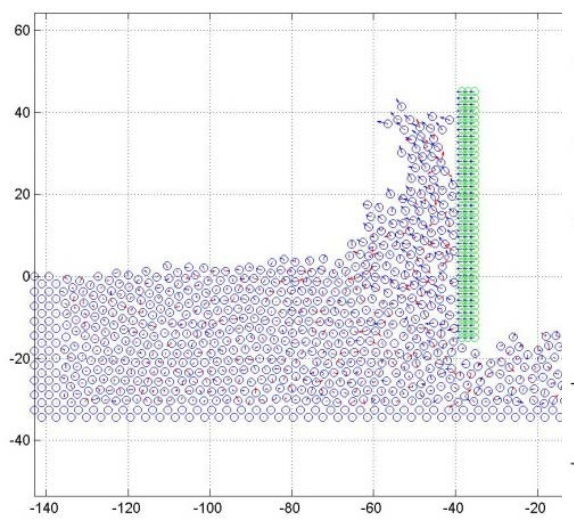

(b)

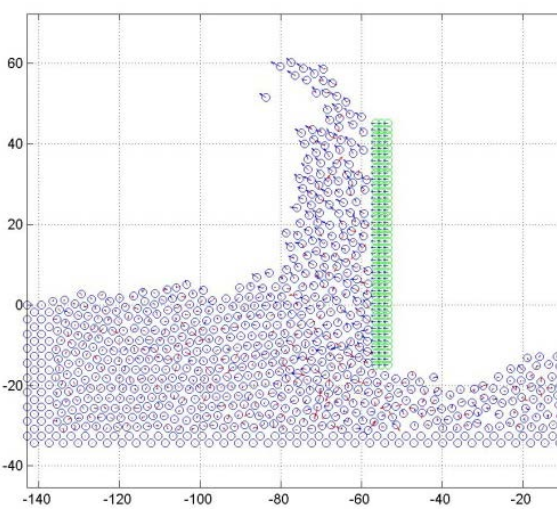

(c)

Fig.2. Simulations for $0^{\circ}$ tool rake angle, depth of cut $15 \AA$ at (a) 132 ps, (b) 264ps and (c) 363 ps of the analysis

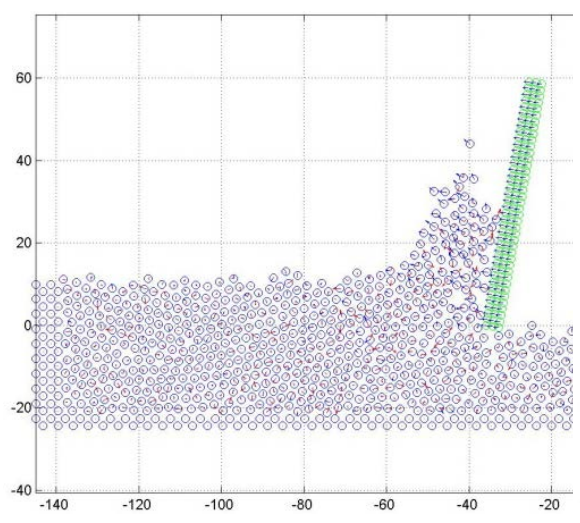

(a)

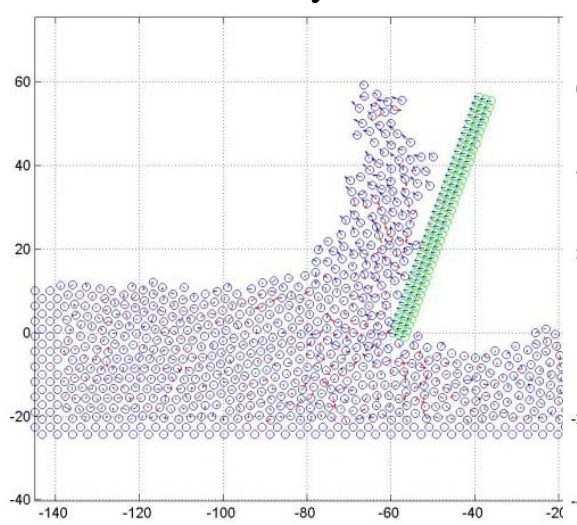

(b)

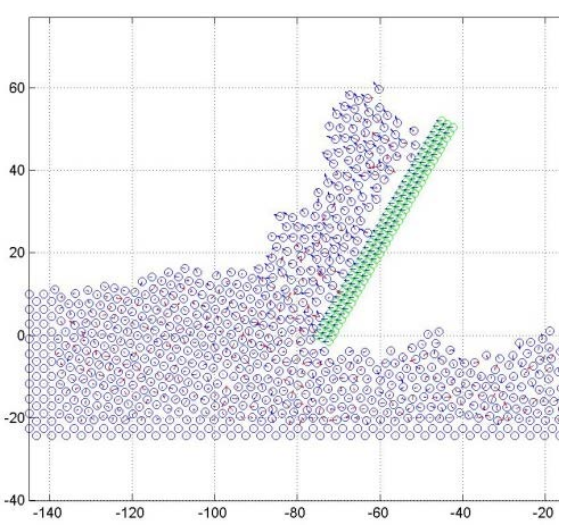

(c)

Fig.3. Simulations for depth of cut $10 \AA$ and tool rake angle of (a) $10^{\circ}$, (b) $20^{\circ}$ and (c) $30^{\circ}$

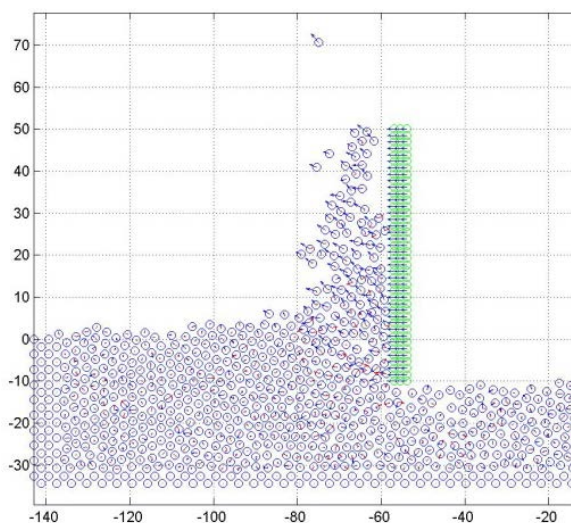

(a)

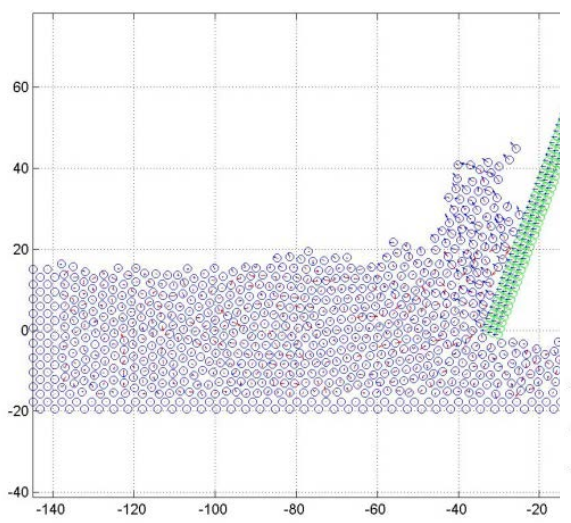

(b)

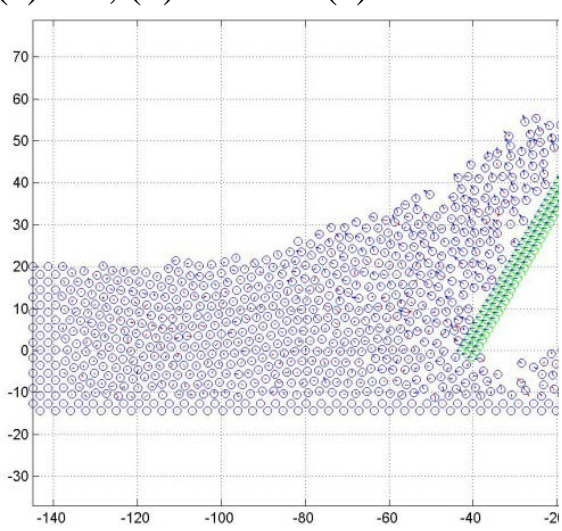

(c)

Fig.4. Simulations for (a) depth of cut $10 \AA$, rake angle $0^{\circ}$, (b) depth of cut $15 \AA$, rake angle $20^{\circ}$ and (c) depth of cut $20 \AA$, rake angle $30^{\circ}$ 


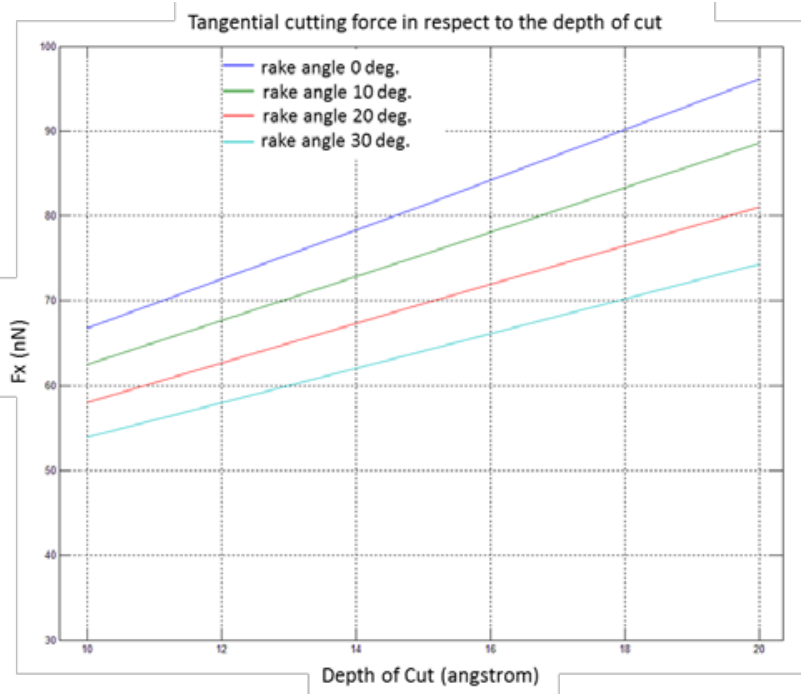

(a)

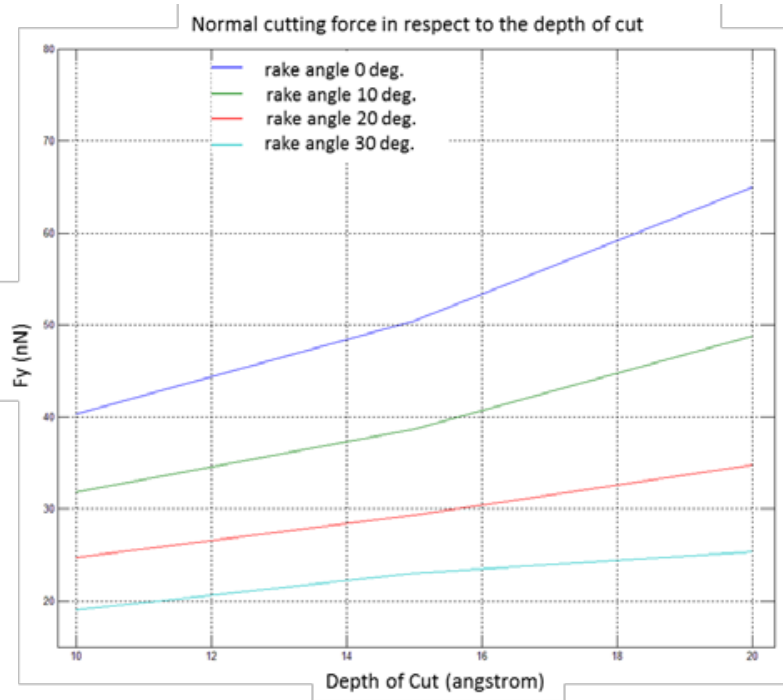

(b)

Fig. 5. Diagrams of (a) $F_{x}$ and (b) $F_{y}$, for various depths of cut, for increasing rake angle.

An increase in the rake angle results in an improvement of surface quality and a decrease of the cutting forces. However, a further increase in the rake angle, more than $20-30^{\circ}$, induces a rather minimal difference in the surface quality and the cutting forces exerted on the workpiece, see Table 3.

Table 3. Numerical results for various model cases

\begin{tabular}{ccccc}
\hline Rake angle & Depth of cut $(\AA)$ & $\mathrm{F}_{\mathrm{x}}(\mathrm{nN})$ & $\mathrm{F}_{\mathrm{y}}(\mathrm{nN})$ & $\mathrm{F}_{\mathrm{y}} / \mathrm{F}_{\mathrm{x}}$ \\
\hline \multirow{3}{*}{$0^{0}$} & 10 & 66.8 & 40.3 & 0,603 \\
& 15 & 81.2 & 50.4 & 0.621 \\
& 20 & 96.1 & 65 & 0.676 \\
\hline \multirow{3}{*}{$10^{0}$} & 10 & 62.5 & 31.8 & 0.509 \\
& 15 & 75.4 & 38.7 & 0.513 \\
& 20 & 88.6 & 48.8 & 0.551 \\
\hline \multirow{3}{*}{$20^{\circ}$} & 10 & 58 & 24.7 & 0.426 \\
& 15 & 69.7 & 29.3 & 0.420 \\
\hline \multirow{3}{*}{$30^{\circ}$} & 20 & 81 & 34.8 & 0.429 \\
& 10 & 53.9 & 19 & 0.359 \\
& 15 & 64.1 & 23 & 0.359 \\
\hline
\end{tabular}

\subsubsection{Effect of cutting tool morphology and cutting speed}

Tool shape is another key factor that affects chip formation. In cases where a cutting tool with a different morphology is used, a more uniform surface, fewer voids in the workpiece material and the chip, lower friction between the tool and the workpiece and finally better quality of the machined surface are obtained due to the better interaction between the tool and workpiece atoms. Furthermore, in a case with a greater number of atoms, it was also found that using a tool of bigger size can produce even better results regarding the chip formation and lower radically the number of voids, see Fig. 6 (a) and (b). This result is considered rather invaluable and it needs to be carefully investigated in a future study in order to determine tool geometries that facilitate the machining process by inducing minimal disorder in the workpiece material and prolongs tool life. By examining cases with a higher cutting speed and a depth of cut of $10 \AA$, it is found that the cutting speed affects the chip formation mechanism to great extent, see Fig. 7 (a)-(d). The workpiece atoms tend to achieve a higher speed and acceleration, the distances between them increase, resulting in more voids and producing a rougher surface with a comparatively lower surface quality. Moreover, the cutting forces increase with an increase in the cutting speed, see Fig. 8 (a) and (b). 


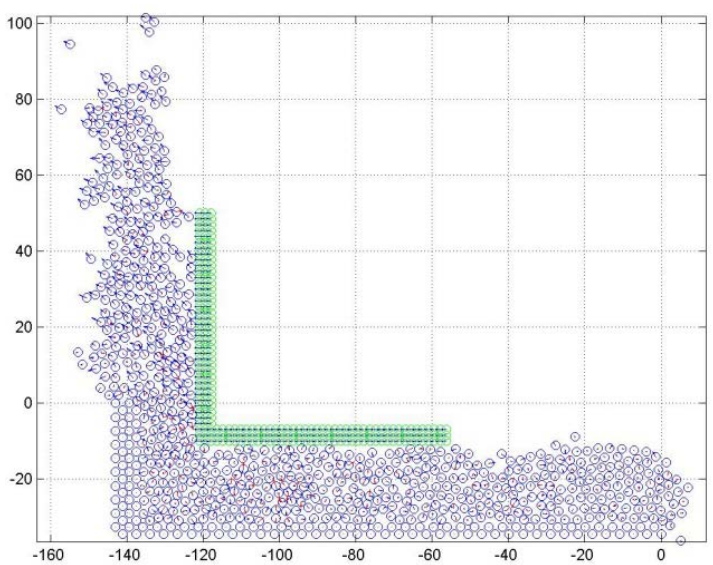

(a)

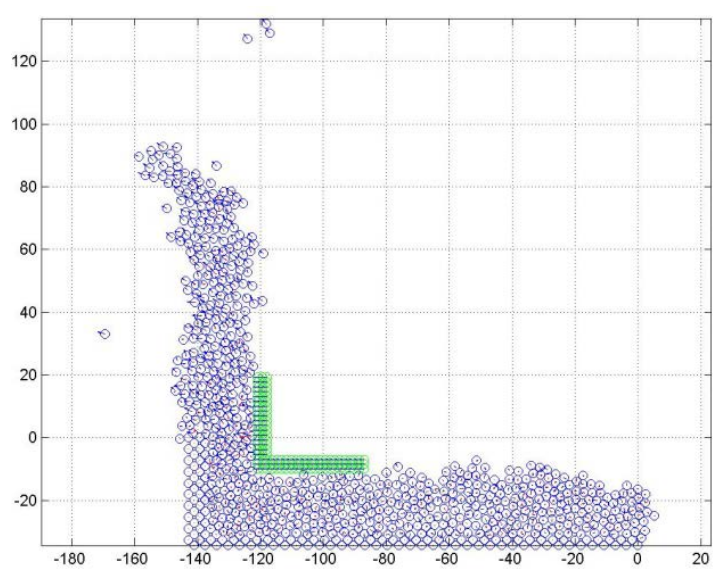

(b)

Fig. 6. Simulations with different tools

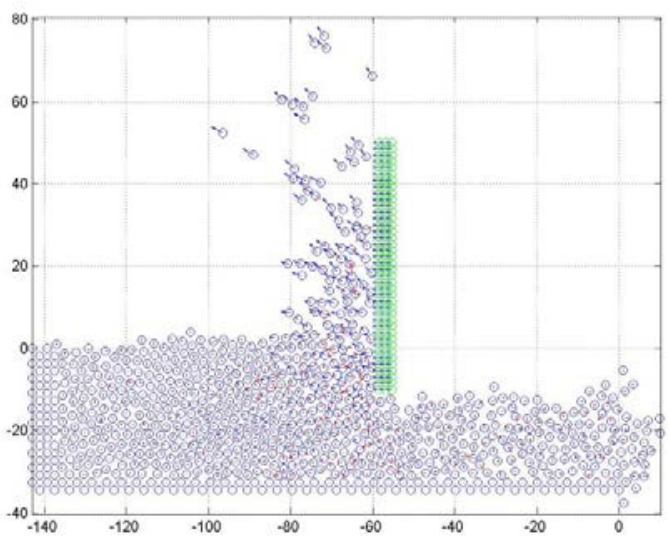

(a)

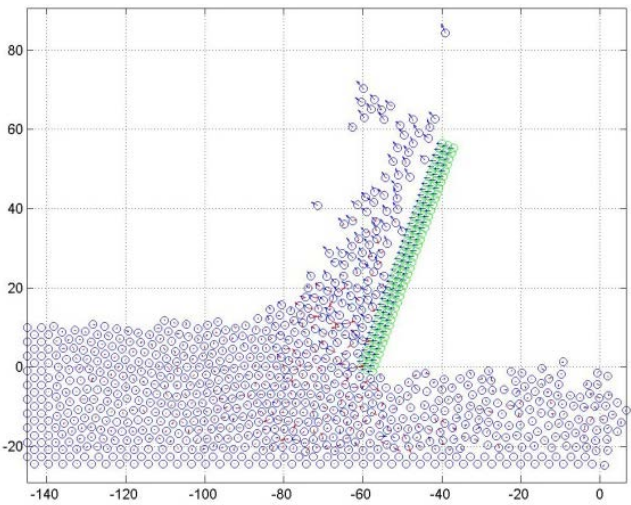

(c)

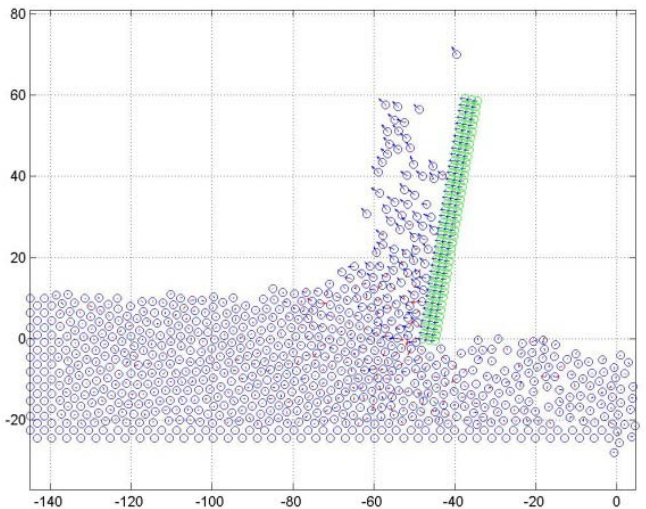

(b)

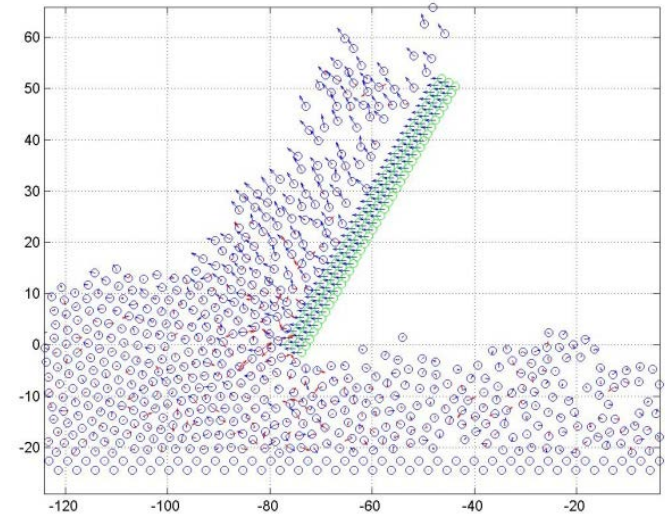

(d)

Fig.7. Simulations with cutting speed $246 \mathrm{~m} / \mathrm{s}$, depth of cut $10 \AA$ and rake angle (a) $0^{\circ}$, (b) $10^{\circ}$, (c) $20^{\circ}$ and (d) $30^{\circ}$

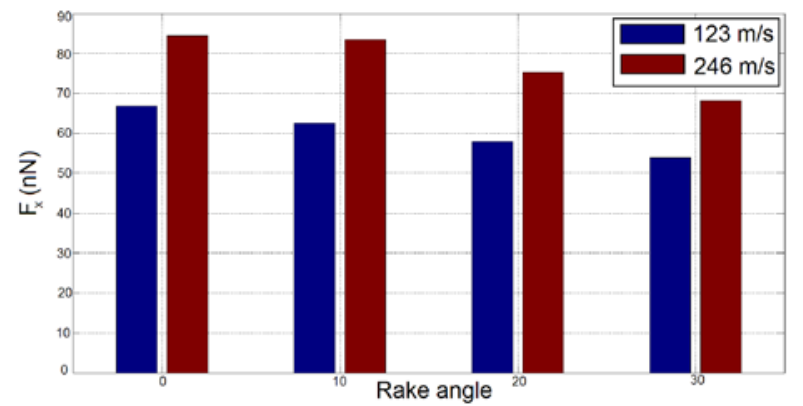

(a)

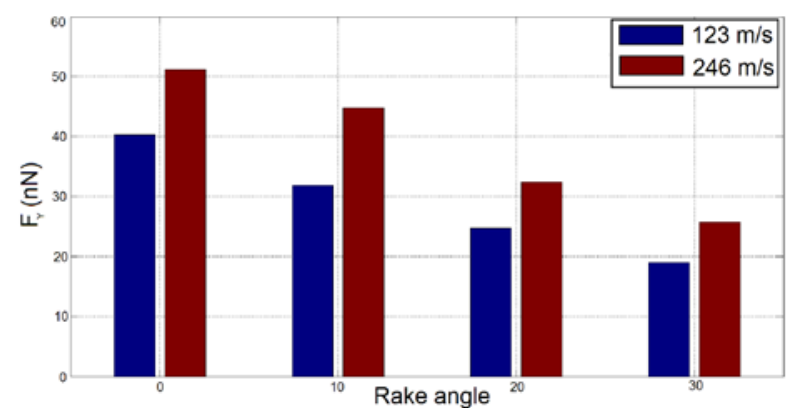

(b)

Fig. 8. Comparison of (a) $F_{x}$ and (b) $F_{y}$ for all rake angles, for cutting speeds $123 \mathrm{~m} / \mathrm{s}$ and $246 \mathrm{~m} / \mathrm{s}$ 


\subsubsection{Effect of potential function}

In cases conducted using the Morse potential instead of the Lennard-Jones potential, only slightly different results in terms of chip formation and surface quality were obtained. It is worth noting that the Morse potential produced lower cutting forces values (see Fig.9 (a) and (b)). The overestimation of cutting forces when the Lennard-Jones potential function is employed needs to be carefully taken into consideration, as a more realistic estimation of cutting forces is desirable; this can only be determined by an experimental procedure. However, chip morphology appears to be more consistent with the anticipated chip morphology from micro-machining experiments in the case of Morse rather than Lennard-Jones potential.

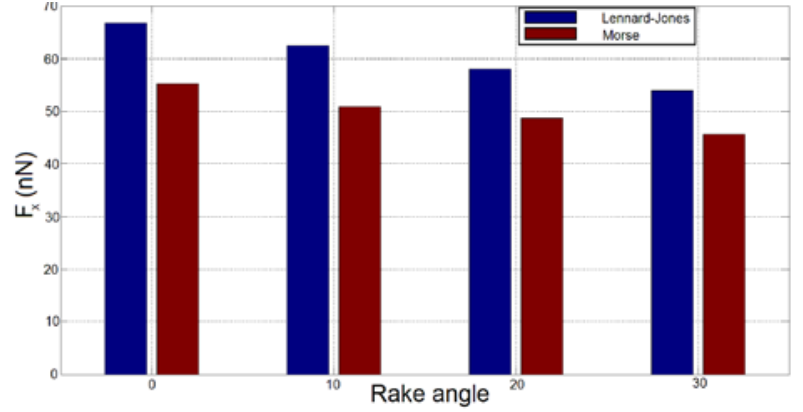

(a)

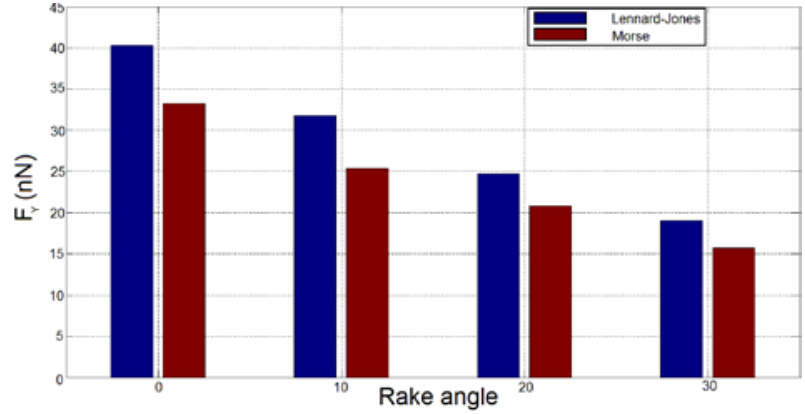

(b)

Fig. 9. Comparison of (a) $F_{x}$ and (b) $F_{y}$, for all rake angles, for models using Lennard-Jones and Morse potential

\subsection{Cases with thermostat atoms}

In the second part of this investigation, two layers of thermostat atoms were added in the MD model and the effect of several parameters in the chip formation mechanism and the temperature profile along selected areas of the workpiece were investigated.

\subsubsection{Temperature profile}

As far as the temperature profile in the workpiece is concerned, the maximum temperature in the workpiece was about $53 \mathrm{~K}$ lower in cases with thermostat atoms and a depth of cut of $10 \AA$ compared to cases with the same depth of cut but without thermostat atoms. The temperature in the workpiece increases also gradually with an increase in the depth of cut but remains almost unchanged in the case where the Lennard-Jones potential is used. Increasing the depth of cut, the temperature increases, because the cutting tool encounters even more atoms, which obtain a greater velocity.

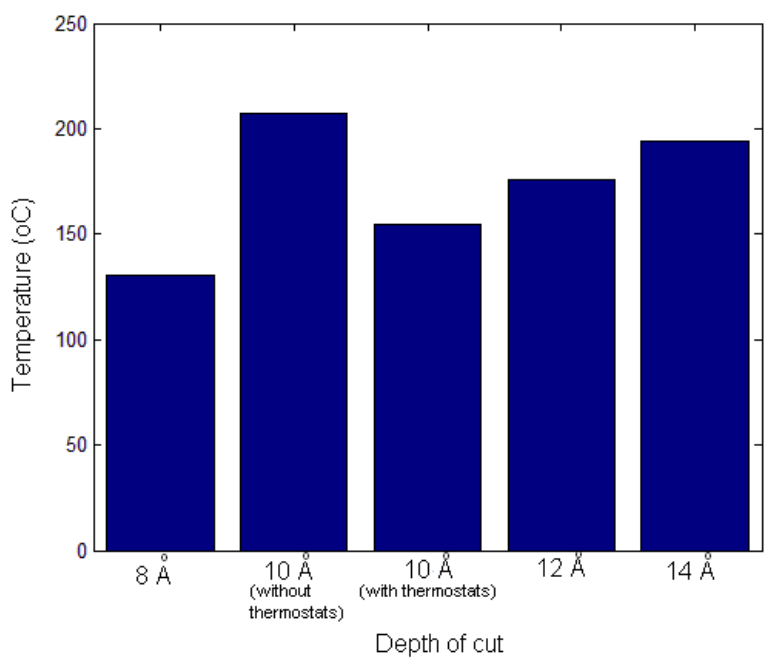

Fig. 10. Maximum temperature in the workpiece 
Thus, this increase in the kinetic energy results to an increase in the workpiece temperature in the areas of the workpiece that are primarily affected by the movement of the cutting tool.This local increase of kinetic energy, due to the more intense interactions of the workpiece and tool atoms leads to greater deformation, more voids and inhomogeneities in the workpiece material in these areas. In Fig. 10, the maximum temperature observed during various simulation cases is depicted.

\subsubsection{Chip formation mechanism and cutting forces}

It was found that the use of thermostat atoms, in the examined cases, does not contribute to major differences in the chip formation mechanism and a few variations can be observed regarding the machined surface quality. A slight decrease in terms of cutting forces values, namely about 5\%, was observed in cases with thermostat atoms; this can be attributed to the lower kinetic energy in the workpiece in these cases.

\section{Conclusions}

In this paper, an orthogonal Molecular Dynamics model was used for the simulation of the nanoscale cutting process of a copper workpiece using a rigid single-crystal diamond tool. The effect of various parameters such as the depth of cut, rake angle, cutting speed, potential function and the use of a thermostat atoms layer in the chip formation mechanism, the cutting forces and the workpiece temperature was investigated.

- The chip formation mechanism was shown to be dependent of the majority of the parameters examined. Thus, a small depth of cut, a greater rake angle, a lower cutting speed, a tool which is in contact with more atoms of the workpiece and the use of Morse potential instead of LennardJones potential produce generally better results, as the chip looks more compact. The use of thermostat atoms in several cases did not significantly alter the produced chip morphology.

- The cutting forces were found to increase with an increase of the depth of cut, a decrease of the rake angle, an increase of the cutting speed and the use of Lennard-Jones potential instead of Morse potential. The use of thermostat atoms did not produce a significant change of the cutting forces.

- The temperature of the workpiece was higher in simulations with greater depth of cut and no thermostat atoms. On the contrary, the use of thermostat atoms enabled a significant reduction of the temperature in the workpiece. Thus, the use of the thermostat atoms layers allowed the effective regulation of temperature within the workpiece, as it was intended and so the use of thermostat atoms is shown to be essential in every MD nano-machining simulation. Finally, the use of a different potential function did not show any notable change in the temperature.

Consequently, the cases which were conducted allowed the identification of the significance of several basic parameters in the nano-cutting process. These conclusions will be of great importance in further investigations of the chip formation mechanism and other physical phenomena involved in nano-cutting.

\section{Acknowledgement}

The authors would like to thank the anonymous referees for constructive comments on earlier version of this paper. 


\section{References}

Alder, B.J. \& Wainwright, T.E. (1957) Phase transition for a hard sphere model. The Journal of Chemical Physics, 27(6), 1208-1209.

Belak, J. \& Stowers, I.F. (1990) A molecular dynamics model of the orthogonal cutting process. in Proceedings of the ASPE Annual Conference, Rochester, New York, 100-104.

Belak, J., Boercker, D.B. \& Stowers, I.F. (1993) Simulation of nanometre-scale deformation of metallic and ceramic. MRS Bulletin, 18(5), 55-60.

Cai M.B., Li X.P. \& Rahman M. (2007) Study of the temperature and stress in nanoscale ductile mode cutting of silicon using molecular dynamics simulation. Journal of Materials Processing Technology, 192-193, 607-612.

Dziedzic J., Rychcik-Leyk M. \& Rybicki J. (2008) Degradation of a nano-cutting tool: An MD simulation. Journal of Non-Crystalline Solids, 354, 4309-4315.

Girifalco, L.A. \& Weizer, V.G. (1959) Application of the Morse potential function to cubic materials. Physics Review, 114(3), 687-690.

Ikawa, N., Shimada, S., Tanaka, H. \& Ohmori, G. (1991) An Atomistic Analysis of Nanometric Chip Removal as Affected by Tool-Work Interaction in Diamond Turning. CIRP Annals - Manufacturing Technology, 40(1), 551-554.

Inamura, T., Takezawa, N. \& Taniguchi, N. (1992) Atomic-Scale Cutting in a Computer Using Crystal Models of Copper and Diamond. CIRP Annals - Manufacturing Technology, 41(1), 121-124.

Isono Y. \& Tanaka T. (1997) Three dimensional molecular dynamics simulation of atomic scale precision processing using a pin tool. JSME International Journal Series A Solid Mechanics and Material Engineering, 40(3), 211-218.

Kim, J. D. \& Moon, C.H. (1995) A study on the Cutting Mechanism of Microcutting using Molecular Dynamics. International Journal of Advanced Manufacturing Technology, 11, 319-324.

Komanduri R. \& Raff L.M. (2001) A review on the molecular dynamics simulation of machining at the atomic scale. Proceedings of the Institution of Mechanical Engineers, Part B, Journal of Engineering Manufacture, 215, 1639 - 1672.

Komanduri, R., Chandrasekaran, N. \& Raff, L.M. (1998) Effect of tool geometry in nanometric cutting: a molecular dynamics simulation approach. Wear, 219(1), 84-97.

Komanduri, R., Chandrasekaran, N. \& Raff, L.M. (2000) M.D. Simulation of nanometric cutting of single crystal aluminum - effect of crystal orientation and direction of cutting. Wear, 242(1-2), 6088.

Li J., Fang Q., Zhang L \& Liu Y. (2015) The effect of rough surface on nanoscale high speed grinding by a molecular dynamics simulation. Computational Materials Science, 98, 252-262.

Maekawa, K. \& Itoh, A. (1995) Friction and tool wear in nano-scale machining- a molecular dynamics approach. Wear, 188, 115-122.

Markopoulos A.P. (2013) Finite Element Method in Machining Processes. London: Springer

Markopoulos A.P. \& Manolakos D.E. (2014) Modeling of Micromachining. in: Davim, J.P (Ed), Modern Mechanical Engineering, Springer-Verlag, Berlin Heidelberg, 285-323.

Metropolis N., Rosenbluth A.W., Rosenbluth M.N., Teller A.H. \& Teller E. (1953) Equation of State Calculations by Fast Computing Machines. The Journal of Chemical Physics, 21(6), 1087-1092

Oluwajobi A.O. \& Chen X. (2010) The fundamentals of modelling abrasive machining using molecular dynamics. International Journal of Abrasive Technology, 3(4), 354-381.

Pei, Q.X., Lu, C., Fang, F.Z. \& Wu, H. (2006) Nanometric cutting of copper: A molecular dynamics study. Computational Materials Science, 37, 434-441.

Rentsch R. \& Inasaki I. (1994) Molecular Dynamics Simulation for Abrasive Processes. CIRP AnnalsManufacturing Technology, 43(1), 327-330.

Romero P.A., Anciaux G., Molinari A. \& Molinari J.F. (2013) Insights into the thermo-mechanics of orthogonal nanometric Machining. Computational Materials Science, 72, 116-126.

Romero P.A., Järvi T.T., Beckmann N., Mrovec M. \& Moseler M. (2014) Coarse Graining and 
Localized Plasticity between Sliding Nanocrystalline Metals. Physical Review Letters, 113, 036101. Rapaport, D.C. (2004). The Art of Molecular Dynamics Simulation. Cambridge University Press.

Ye, Y.Y., Biswas, R., Morris, J.R., Bastawros, A. \& Chandra A. (2003) Molecular dynamics simulation of nanoscale machining of copper. Nanotechnology, 14, 390-396.

Zhu, P.-Z., Qiu, C., Fang, F.-Z., Yuan, D.-D. and Shen, X.C. (2014) Molecular dynamics simulations of nanometric cutting mechanisms of amorphous alloy. Applied Surface Science, 317, 432-442. 